\title{
Estimation of Moments of Digitized Objects with Fuzzy Borders
}

\author{
Nataša Sladoje and Joakim Lindblad \\ Centre for Image Analysis, \\ Swedish University of Agricultural Sciences and Uppsala University, Uppsala, Sweden \\ \{natasa, joakim\}@cb.uu.se
}

\begin{abstract}
Error bounds for estimation of moments from a fuzzy representation of a shape are derived, and compared with estimations from a crisp representation. It is shown that a fuzzy membership function based on the pixel area coverage provides higher accuracy of the estimates, compared to binary Gauss digitization at the same spatial image resolution. Theoretical results are confirmed by a statistical study of disks and squares, where the moments of the shape, up to order two, are estimated from its fuzzy discrete representation. The errors of the estimates decrease both with increased size of a shape (spatial resolution) and increased membership resolution (number of available grey-levels).
\end{abstract}

Keywords: Fuzzy sets, moments, accuracy, multigrid resolution.

\section{Introduction}

Fuzziness is a natural outcome of most image processing techniques. It is an intrinsic quality of images and often provides important information about the image [15. Fuzzy membership of a point reflects the level to which that point fulfils certain criteria to be a member of a set. The process of converting an input image to a fuzzy set by indicating, for each pixel, the degree of membership to the object, is referred to as fuzzy segmentation. Different imaging devices produce grey-level images with different underlying interpretations; a knowledge about such specific properties of the image is preferably incorporated into the fuzzy segmentation method that is applied. In practise, area coverage related memberships often result from the imaging process. Brightness of a pixel is proportional to the part of its area covered by the observed object, and greylevels can therefore directly be used for defining membership values of a fuzzy object. Most image points are assigned memberships (close to) zero or (close to) one, so they can easily be classified as either object or background. Some of the points, often located around the border of an object, are assigned memberships between zero and one, according to the extent of their membership to the object. A segmentation resulting in a digital object with a fuzzy border is usually easier to perform than a crisp one.

In our previous work, it is shown that the precision of estimates of various properties of a continuous original shape is highly improved when derived from a 
fuzzy, instead of a crisp representation of a continuous object [4. In this paper, we analyse the accuracy of the estimation of moments, when they are calculated from a fuzzy representation of a shape. We show that the order of magnitude of the error can be reduced if the estimation is based on a fuzzy discrete shape representation; a fuzzy approach can be an alternative to increasing the resolution of the image.

\section{Moments Estimated from a Crisp Discrete Set}

Definition 1. The $(p, q)$-moment of a planar crisp set $S$ in the Euclidean plane equipped with the Cartesian xy-coordinate system is defined by

$$
m_{p, q}(S)=\iint_{S} x^{p} y^{q} d x d y,
$$

for integers $p, q \geq 0$. The moment $m_{p, q}(S)$ has the order $p+q$.

If the set $S$ is inscribed into an integer grid and digitized, instead of the moments $m_{p, q}(S)$, the moments of the digitization $\mathcal{D}(S)$ are available.

Definition 2. The discrete moment $\tilde{m}_{p, q}(S)$ of a crisp set $S$ is defined by

$$
\tilde{m}_{p, q}(S)=\sum_{(i, j) \in \mathcal{D}(S)} i^{p} j^{q},
$$

where $\mathcal{D}(S)$ is the set of integer grid points contained in the set $S$.

The concept of moments is introduced in image analysis by $\mathrm{Hu}$ [2. Several features of a shape can be calculated from a sufficient number of its moments. In fact, a shape can be recovered from an appropriately chosen set of its moments. If continuous moments are replaced by the their discrete counterparts, more or less good estimates of the observed features of a continuous shape can be obtained.

One of the main disadvantages of using moments for a description of a shape is that they are time consuming to compute; the problem increases with the increase of the image resolution. On the other hand, the accuracy of the estimations of the continuous shape features from the corresponding discrete moments increases with the increase of the image resolution. Consequently, it is desirable to use the information about the expected error of a moment estimation at a certain resolution, to get the best accuracy with the lowest possible computational effort, as discussed in [36]. In this paper, we study the possibility of using a fuzzy representation of a shape in order to increase the accuracy of moments estimation at a given spatial resolution. We use the results proved in [3], stated below, to derive error bounds for moments estimated from fuzzy sets.

Theorem 1. The moments of a convex set $S$, with a boundary consisting of a finite number of $C^{3}$ arcs, digitized in a grid with resolution $r$ (the number of grid points per unit), can be estimated from a set $r S=\{(r x, r y) \mid(x, y) \in S\}$ by

$$
m_{p, q}(S)=\frac{1}{r^{p+q+2}} \tilde{m}_{p, q}(r S)+\mathcal{O}\left(\frac{1}{r}\right),
$$


for $p+q \leq 2$. If $S$ is a 3-smooth convex set, its moments can be estimated by

$$
m_{p, q}(S)=\frac{1}{r^{p+q+2}} \tilde{m}_{p, q}(r S)+\mathcal{O}\left(\frac{1}{r^{\frac{15}{11}-\varepsilon}}\right) .
$$

Remark 1: A planar 3-smooth convex set is a convex set in the Euclidean plane whose boundary consists of a finite number of arcs having continuous third order derivatives and a positive curvature at every point, except the end points of the arcs. These conditions exclude the existence of straight boundary segments. Remark 2: The set $r S=\{(r x, r y) \mid(x, y) \in S\}$ is a dilation of the continuous set $S$ by the grid resolution $r$.

\section{Moments Estimated from a Fuzzy Discrete Set}

Fuzzy sets are defined by Zadeh [7.

Definition 3. A fuzzy subset $S$ of a reference set $X$ is a set of ordered pairs $S=\left\{\left(x, \mu_{S}(x)\right) \mid x \in X\right\}$, where $\mu_{S}: X \rightarrow[0,1]$ is the membership function of $S$ in $X$.

We consider discrete fuzzy sets as representations of discretized continuous crisp shapes. Fuzzy moments and the centre of gravity of a fuzzy set are among the first defined fuzzy concepts.

Definition 4. The discrete fuzzy moment $f \tilde{m}_{p, q}(S)$ of a fuzzy set $S$ of a reference set $X \subset Z^{2}$ is

$$
f \tilde{m}_{p, q}(S)=\sum_{(i, j) \in X} \mu_{S}(i, j) i^{p} j^{q}
$$

where $\mu_{S}(i, j)$ is the membership function of $S$.

In the following, $c \tilde{m}_{p, q}(S)$ denotes a crisp discrete moment of a set $S$.

We analyze and compare the error bounds for the two approaches, i.e., when crisp and fuzzy discrete moments, respectively, are used in the estimation of the moments of a continuous shape.

The question we would like to answer is: If we are not able to increase the spatial resolution of an image in order to achieve an increased accuracy of the shape moment estimation, how much do we gain if we use a fuzzy representation of the object instead of a crisp one?

To answer this question, we observe a pixel $P_{f}$ in a grid with a spatial resolution $r_{s}$ and membership resolution $r_{f}^{2}$ (the number of grey-levels per unit, here equal to the number of grey-levels available), and compare it with a block $P_{c}$ of $r_{f} \times r_{f}$ pixels in a grid of a spatial resolution $r_{s} r_{f}$ and membership resolution 1 . Fuzzy membership of a pixel $P_{f}$ is assigned according to its area coverage, and is approximated by $k / r_{f}^{2}$. This corresponds to the number of sub-pixels, denoted by $k$ (out of $r_{f}^{2}$ ), within the pixel $P_{f}$, which are covered by the observed continuous 
crisp shape $S$. For a sub-sampling factor $r_{f}$, the number of possible grey-levels is $r_{f}^{2}$, which requires a pixel depth of $2 \log _{2}\left(r_{f}\right)$ for representation. In the alternative approach, we consider an $r_{f}$ times dilated crisp representation of a shape. For the dilated shape, the pixels within $P_{c} \cap S$ correspond to the sub-pixels within $P_{f}$, covered by $S$. Their number is equal to $k$, for $k \in\left\{0,1, \ldots, r_{f}^{2}\right\}$.

We assume that the size of a pixel is equal to 1 . Consequently, the size of $P_{c}$ is equal to $r_{f} \times r_{f}$. The coordinates of a pixel $P_{f}$ are $\left(r_{s} x, r_{s} y\right)$, while the coordinates of the pixels within a block $P_{c}$ can be expressed as

$$
\left(r_{s} r_{f} x-\frac{r_{f}}{2}+\frac{1}{2}+i, r_{s} r_{f} y-\frac{r_{f}}{2}+\frac{1}{2}+j\right),
$$

where $i, j \in\{0,1, \ldots, r-1\}$.

\subsection{Zero-Order Moment Estimation}

The contribution of the pixel $P_{f}$ to the fuzzy discrete moment $f \tilde{m}_{0,0}\left(r_{s} S\right)$ is

$$
f \tilde{m}_{0,0}^{(1)}\left(r_{s} S\right)=\frac{k}{r_{f}^{2}}
$$

The upper-index (1) denotes the contribution of one fuzzy pixel and one block of crisp pixels, respectively.

Alternatively, if the grid resolution $r_{s}$ is increased $r_{f}$ times, there are $k$, out of $r_{f}^{2}$, pixels having their centroids within the continuous crisp shape and contributing to the zero-order crisp moment. The contribution of $P_{c}$ to the moment $c \tilde{m}_{0,0}\left(r_{s} r_{f} S\right)$ is

$$
c \tilde{m}_{0,0}^{(1)}\left(r_{s} r_{f} S\right)=k .
$$

Considering all $r_{s} \times r_{s}$ fuzzy pixels/crisp blocks in the image, we derive the following relation between the crisp and the fuzzy discrete moments of the observed (discrete) shape:

$$
c \tilde{m}_{0,0}\left(r_{s} r_{f} S\right)=r_{f}^{2} f \tilde{m}_{0,0}\left(r_{s} S\right) .
$$

By using (3) and Theorem 1] it follows that the zero-order moment of a continuous convex shape $S$ can be estimated by

$$
m_{0,0}(S)=\frac{1}{r_{s}^{2} r_{f}^{2}} c \tilde{m}_{0,0}\left(r_{s} r_{f} S\right)+\mathcal{O}\left(\frac{1}{r_{s} r_{f}}\right)=\frac{1}{r_{s}^{2}} f \tilde{m}_{0,0}\left(r_{s} S\right)+\mathcal{O}\left(\frac{1}{r_{s} r_{f}}\right),
$$

while the accuracy of the estimation of the $m_{0,0}(S)$ moment of a 3-smooth convex shape $S$ can be expressed by

$$
m_{0,0}(S)=\frac{1}{r_{s}^{2}} f \tilde{m}_{p, q}\left(r_{s} S\right)+\mathcal{O}\left(\frac{1}{\left(r_{s} r_{f}\right)^{\frac{15}{11}-\varepsilon}}\right) .
$$

Thus, we conclude that using $r_{f}^{2}$ pixel membership values provides the same accuracy of $m_{0,0}(S)$ estimation as increasing the (crisp) image spatial resolution $r_{f}$ times. 


\subsection{First-Order Moments Estimation}

Moments of order higher than zero are position variant. At low resolutions, the accuracy of the estimation cannot be fully preserved if the spatial resolution is $r_{f}$ times decreased, and fuzzy resolution $r_{f}^{2}$ is used instead. However, the asymptotic expressions that we derive show that such a behaviour is expected if the spatial resolution is high enough.

The contribution of the observed pixel $P_{f}$ (having coordinates $\left(r_{s} x, r_{s} y\right)$ ) to the moment $f \tilde{m}_{1,0}\left(r_{s} S\right)$ is

$$
f \tilde{m}_{1,0}^{(1)}\left(r_{s} S\right)=r_{s} x \frac{k}{r_{f}^{2}} .
$$

Note that only the number of covered sub-pixels is taken into account, and not their coordinates.

On the other hand, if the grid resolution $r_{s}$ is increased $r_{f}$ times, there are $k$ out of $r_{f}^{2}$ pixels having their centroids within the continuous crisp shape and therefore contributing to the first-order crisp moment. The contribution of $P_{c}$ to the moment $c \tilde{m}_{1,0}\left(r_{s} r_{f} S\right)$ is

$$
c \tilde{m}_{1,0}^{(1)}\left(r_{s} r_{f} S\right)=\left(r_{s} r_{f} x-\frac{r_{f}}{2}+\frac{1}{2}\right) k+\sum i,
$$

where $i$ takes $k$ values from the set $\{0,1, \ldots, r-1\}$, depending on the position of the $k$ covered pixels within $P_{c}$.

It is easy to conclude that in the case when $k=r_{f}^{2}$, i.e., when all pixels within the block $P_{c}$ are covered by $S$, and, equivalently, the membership value of $P_{f}$ to the observed shape is equal to 1 ,

$$
c \tilde{m}_{1,0}^{(1)}\left(r_{s} r_{f} S\right)=r_{f}^{3} f \tilde{m}_{1,0}^{(1)}\left(r_{s} S\right) .
$$

Under the assumption made about the fuzzy representation of the observed convex shape, $\mathcal{O}\left(r_{s}^{2}\right)$ of the image points (all of the inner pixels) are of this type.

The pixels on the border of a shape have membership values between 0 and 1 . The biggest difference between the contributions of $P_{f}$ to the fuzzy moment and of the block $P_{c}$ to the crisp moment appears when one half of a pixel/block (left or right part) is covered:

$$
c \tilde{m}_{1,0}^{(1)}\left(r_{s} r_{f} S\right)=r_{f}^{3} f \tilde{m}_{1,0}^{(1)}\left(r_{s} S\right)+\mathcal{O}\left(r_{f}^{3}\right) .
$$

We assume that there are $\mathcal{O}\left(r_{s}\right)$ pixels of this type in the observed fuzzy image.

Considering (6) and (77), we derive the following relation between the crisp and the fuzzy discrete moments of an observed (discrete) shape:

$$
c \tilde{m}_{1,0}\left(r_{s} r_{f} S\right)=r_{f}^{3} f \tilde{m}_{1,0}\left(r_{s} S\right)+\mathcal{O}\left(r_{s} r_{f}^{3}\right) .
$$

By using (8) and Theorem [1, it follows that the first-order moment of a continuous convex shape $S$ can be estimated by

$$
m_{1,0}(S)=\frac{1}{r_{s}^{3}} f \tilde{m}_{1,0}\left(r_{s} S\right)+\mathcal{O}\left(\frac{1}{r_{s}^{2}}\right)+\mathcal{O}\left(\frac{1}{r_{s} r_{f}}\right) .
$$


We conclude that once the spatial resolution is high enough to fully "exploit" the fuzzy membership values of pixels, i.e., when $r_{s}>C r_{f}$, where $C$ is a constant derived from the asymptotic expression for the error bound, using $r_{f}^{2}$ pixel membership values provides the same accuracy of $m_{1,0}(S)$ estimation as increasing the (crisp) image spatial resolution $r_{f}$ times.

By using (8) and the second part of Theorem 1, for a 3-smooth convex shape $S$ it holds

$$
m_{1,0}(S)=\frac{1}{r_{s}^{3}} f \tilde{m}_{1,0}\left(r_{s} S\right)+\mathcal{O}\left(\frac{1}{\left(r_{s} r_{f}\right)^{\frac{15}{11}-\varepsilon}}\right), \quad \text { for } r_{s}>C r_{f}^{\frac{15}{7}+\varepsilon}
$$

Analogous results follow for the $m_{0,1}(S)$ moment estimation.

\subsection{Second-Order Moments Estimation}

In the derivation of the asymptotic expressions for the estimation of the secondorder moments of a convex shape from its fuzzy representation, we apply similar reasoning as for the first-order moments.

For $\mathcal{O}\left(r_{s}^{2}\right)$ fully covered pixels $P_{f} /$ blocks $P_{c}$, it holds that

$$
c \tilde{m}_{2,0}^{(1)}\left(r_{s} r_{f} S\right)=r_{f}^{4} f \tilde{m}_{2,0}^{(1)}\left(r_{s} S\right)+\mathcal{O}\left(r_{f}^{4}\right)
$$

while for $\mathcal{O}\left(r_{s}\right)$ pixels/blocks on the border of the object, in the worst case (if the right half of the pixel/block is covered by the object) it holds that

$$
c \tilde{m}_{2,0}^{(1)}\left(r_{s} r_{f} S\right)=r_{f}^{4} f \tilde{m}_{2,0}^{(1)}\left(r_{s} S\right)+\mathcal{O}\left(r_{s} r_{f}^{4}\right) .
$$

Considering (11) and (12), for the whole image it follows that

$$
c \tilde{m}_{2,0}\left(r_{s} r_{f} S\right)=r_{f}^{4} f \tilde{m}_{2,0}\left(r_{s} S\right)+\mathcal{O}\left(r_{s}^{2} r_{f}^{4}\right) .
$$

By using (13) and Theorem 1] it follows that the second-order moment of a continuous convex shape $S$ can be estimated by

$$
m_{2,0}(S)=\frac{1}{r_{s}^{4}} f \tilde{m}_{2,0}\left(r_{s} S\right)+\mathcal{O}\left(\frac{1}{r_{s} r_{f}}\right), \text { for } r_{s}>C r_{f} .
$$

As in the case of the first order moment estimation, we conclude that using $r_{f}^{2}$ pixel membership values asymptotically provides the same accuracy of $m_{2,0}(S)$ estimation as increasing the spatial resolution of the (crisp) image $r_{f}$ times.

Similarly, from (13) and the second part of Theorem 11, it follows

$$
m_{2,0}(S)=\frac{1}{r_{s}^{4}} f \tilde{m}_{2,0}\left(r_{s} S\right)+\mathcal{O}\left(\frac{1}{\left(r_{s} r_{f}\right)^{\frac{15}{11}-\varepsilon}}\right), \quad \text { for } r_{s}>C r_{f}{ }^{\frac{15}{7}+\varepsilon}
$$

Analogous results follow for the $m_{1,1}(S)$ and $m_{0,2}(S)$ moments estimation. 


\section{Statistical Study of Squares and Disks}

We perform a statistical study in order to examine the properties of moments estimated at low resolutions. Multigrid resolution is expressed by dilations of the observed objects. Tests are performed for 2, 000 randomly positioned disks (the centres are randomly positioned inside a pixel) for each of a number of observed real-valued radii within the interval $[1,100]$, and for 10,000 randomly positioned squares for each observed real-valued side length within the interval [1,90] (for each size, 100 random centre positions, each with 100 random rotations between 0 and 45 degrees, are considered). Different membership resolutions are used $\left(r_{f} \in\{1,2,4,8,16\}\right)$. Note that the $r_{f}=1$ corresponds to crisp segmentation, and that $r_{f}=16$ gives the upper limit for the membership resolution of 8-bit pixel depth.

For each size of an object, we determine the maximal relative estimation error for moments up to the order two. We present the results for $m_{1,0}$ and $m_{2,0}$ moments estimation, both for squares and for disks, in Figure 1. The estimation
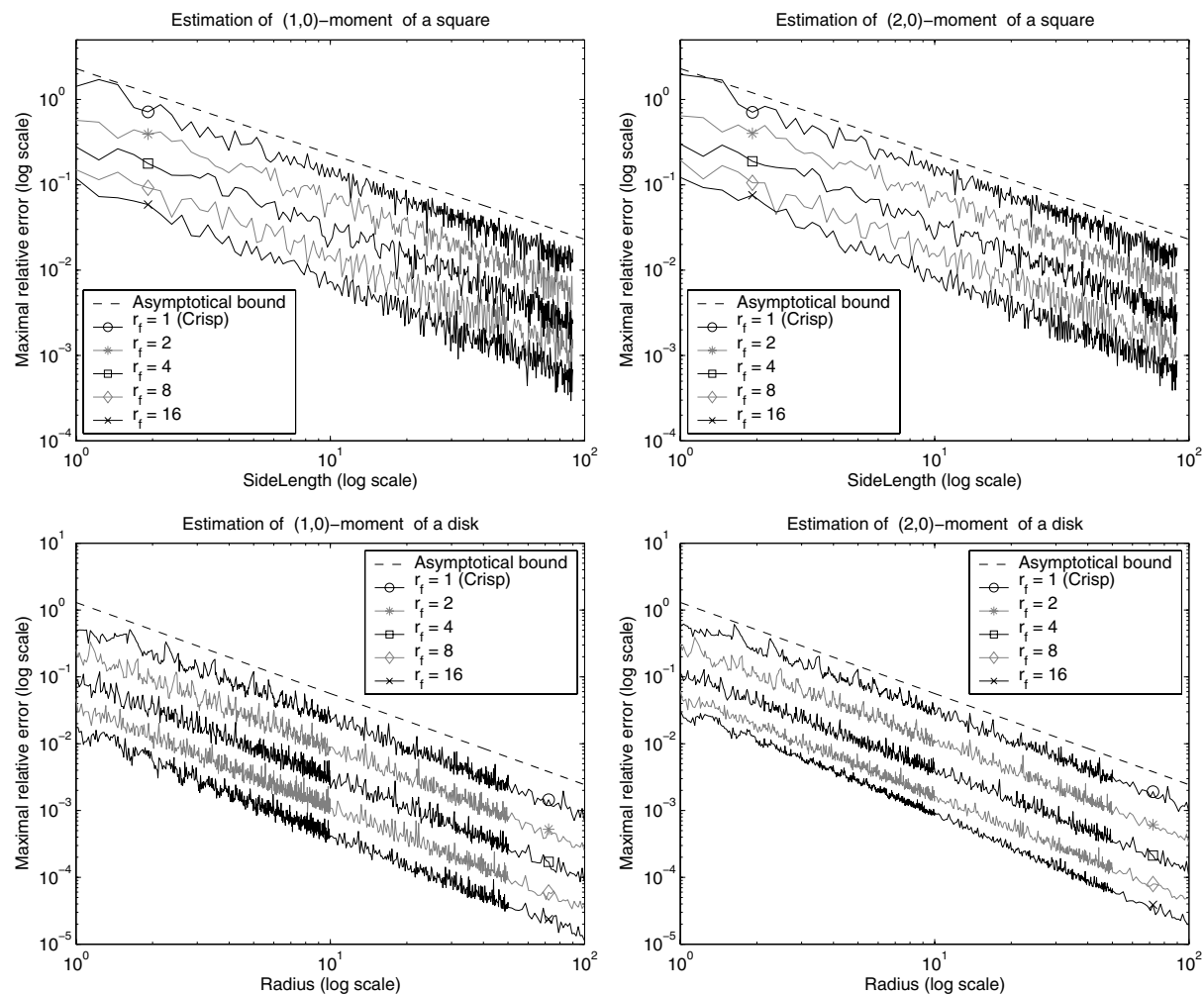

Fig. 1. Error bounds for first and second order moments estimation. Top: Moments estimation of a square. Bottom: Moments estimation of a disk. 
errors for squares show asymptotic behaviour in accordance with expressions (8) and (13). Disks are 3 -smooth convex objects, and the corresponding estimation bounds agree with expressions (10) and (15).

Plots are presented in a logarithmic scale so that the "slopes" of the curves correspond to the order of estimation error, and can be compared with the plotted straight line which has a slope equal to the theoretically derived order of error ( -1 for squares and $-\frac{15}{11}$ for disks). The expected asymptotic behaviour assumes higher errors at low resolutions, which is related to the value of the constant $C$ in (10), (14), and (15). However, for the presented membership resolutions, the plots show accordance with the asymptotic bounds also at low spatial resolutions. The relative position of the curves shows that the estimation error becomes smaller both with the increase of spatial and membership resolution.

\section{Comments and Conclusions}

Derived asymptotic expressions for the estimation of moments of convex shapes from their fuzzy discrete representation show that an increase in membership resolution of an image can be used to achieve the same accuracy of the estimation as an increase of the spatial resolution. Even though the theoretical results guarantee this behaviour only after a certain spatial resolution is reached, the simulations show that it is also present at low resolutions.

By using a fuzzy, instead of a crisp, representation of a shape, which in many cases is easily obtained from the imaging device, significant improvements of the accuracy of moments estimation (and shape features derived from them) are achievable. By fully utilizing an often already existing membership resolution, it is possible to overcome problems of insufficient available spatial resolution.

\section{Acknowledgements}

Prof. Gunilla Borgefors and Doc. Ingela Nyström, both Centre for Image Analysis, Uppsala, Sweden, are gratefully acknowledged for their scientific support.

\section{References}

1. I. Bloch and H. Maître. Fuzzy mathematical morphologies: A comparative study. Pattern Recognition, 28(9):1341-1387, 1995.

2. M. Hu. Visual pattern recognition by moment invariants. IRE Trans. Inform. Theory, 8:179-187, 1962.

3. R. Klette and J. Žunić. Multigrid convergence of calculated features in image analysis. Journal of Mathematical Imaging and Vision, 13:173-191, 2000.

4. N. Sladoje, I. Nyström, and P. Saha. Measurements of digitized objects with fuzzy borders in 2D and 3D. Image and Vision Computing, 23:123-132, 2005.

5. J. K. Udupa and G. J. Grevera. Go digital, go fuzzy. Pattern Recognition Letters, 23:743-754, 2002.

6. J. Žunić and N. Sladoje. Efficiency of characterizing ellipses and ellipsoides by discrete moments. IEEE Trans. on PAMI, 22(4):407-414, 2000.

7. L. Zadeh. Fuzzy sets. Information and Control, 8:338-353, 1965. 